\title{
Review Article \\ Mammalian Cochlear Hair Cell Regeneration and Ribbon Synapse Reformation
}

\author{
Xiaoling Lu, ${ }^{1}$ Yilai Shu, ${ }^{1}$ Mingliang Tang, ${ }^{2,3}$ and Huawei $\mathrm{Li}^{1,4,5,6,7}$ \\ ${ }^{1}$ Otorhinolaryngology Department of Affiliated Eye and ENT Hospital, State Key Laboratory of Medical Neurobiology, \\ Fudan University, Shanghai 200031, China \\ ${ }^{2}$ Key Laboratory for Developmental Genes and Human Disease, Ministry of Education, Institute of Life Sciences, Southeast University, \\ Nanjing 210096, China \\ ${ }^{3}$ Co-Innovation Center of Neuroregeneration, Nantong University, Nantong 226001, China \\ ${ }^{4}$ Institutes of Biomedical Sciences, Fudan University, Shanghai 200032, China \\ ${ }^{5}$ Key Laboratory of Hearing Medicine of NHFPC, Shanghai 200031, China \\ ${ }^{6}$ Shanghai Engineering Research Centre of Cochlear Implant, Shanghai 200031, China \\ ${ }^{7}$ The Institutes of Brain Science and the Collaborative Innovation Center for Brain Science, Fudan University, Shanghai 200032, China
}

Correspondence should be addressed to Mingliang Tang; mingliangtang@seu.edu.cn and Huawei Li; huaweili611@126.com

Received 9 September 2016; Revised 29 November 2016; Accepted 1 December 2016

Academic Editor: Genglin Li

Copyright (C) 2016 Xiaoling Lu et al. This is an open access article distributed under the Creative Commons Attribution License, which permits unrestricted use, distribution, and reproduction in any medium, provided the original work is properly cited.

Hair cells (HCs) are the sensory preceptor cells in the inner ear, which play an important role in hearing and balance. The HCs of organ of Corti are susceptible to noise, ototoxic drugs, and infections, thus resulting in permanent hearing loss. Recent approaches of HCs regeneration provide new directions for finding the treatment of sensor neural deafness. To have normal hearing function, the regenerated HCs must be reinnervated by nerve fibers and reform ribbon synapse with the dendrite of spiral ganglion neuron through nerve regeneration. In this review, we discuss the research progress in $\mathrm{HC}$ regeneration, the synaptic plasticity, and the reinnervation of new regenerated HCs in mammalian inner ear.

\section{Introduction}

Mammalian HCs loss by noise trauma, ototoxic drugs, or infection is a major cause of deafness [1]. HCs in mammalian inner ear, unlike invertebrate animals such as birds and fish, do not undergo spontaneous regeneration, even though vestibular supporting cells (SCs) retain a limited capacity to divide $[2,3]$. There are two approaches of $\mathrm{HC}$ regeneration: (1) direct transdifferentiation of surrounding SCs that directly change cell fate and become HCs and (2) induction of a proliferative response in the SCs which mitotically divide and further differentiate to replace damaged HCs [4-6]. There are various numbers of genes and cell signaling pathways involved in these two mechanisms that remain challenging to understand the molecular mechanism underneath hair cell regeneration. Several studies showed reinnervation of the regenerated HCs after HC regeneration [6-8]. However, innervation of new regenerated HCs still needs to be determined in all kinds of hearing loss.

\section{The Anatomy and Function of the Organ of Corti}

The organ of Corti, also called the spiral organ, is the spiral structure on the basement membrane of the cochlear duct. The sensory epithelium of the organ of Corti is made up of HCs and SCs. HCs, which can be divided into inner HCs and outer HCs, are sensory receptor cells whose mechanically sensitive hair bundles convert mechanical force produced by sound waves into neural impulses. HCs are surrounded by SCs and connected with cochlear nerve fibers by forming synaptic connection. There are several types of SCs, such as pillar cells and phalangeal cells. Pillar cells can be divided into inner and outer pillar cells found in the middle of 


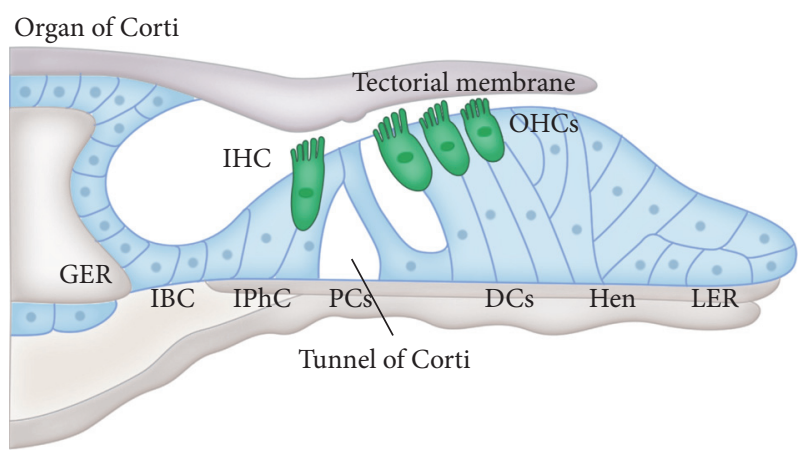

Figure 1: Schematic model of the organ of Corti. IHC: inner hair cell; OHCs: outer hair cells; PCs: inner and outer pillar cells; IPhC: inner phalangeal cell; DCs: Deiters' cells; IBC: inner border cell; Hen: Hensen's cell; GER: greater epithelial ridge; LER: lesser epithelial ridge.

the inner and outer HCs separately. The top and bottom of the inner and outer pillar cells are combined, but the middle of them is separated, forming the two edge sides of the triangular tunnel. In the lateral of inner and outer HCs rows, inner and outer phalangeal cells (also called the Deiters' cells) reside, respectively. The finger like projection of Deiters' cells are tightly connected with the apical of outer pillar cells forming a thin, hard reticular membrane, also called reticular layer. The stereocilium of outer HCs is tightly bounded trough the mesh of reticular layer. The reticular layer constitutes fiber and matrix and is found below the tectorial membrane. HCs are sensory cells, and they do not contain axons and dendrites. Instead, the basolateral surface of HCs form afferent synaptic contacts with the axonal terminals of the eighth nerve and receive efferent contacts from neurons in the brainstem. There are about 25,000 to 30,000 auditory nerve fibers connected with HCs. These fibers originate from bipolar spiral ganglion neurons in the modiolus, whose axonal terminals form synaptic connections with the ribbons at HCs and the dendrite forms connection with cochlear nucleus neuron (Figure 1).

The organ of Corti acts as an auditory receptor. Acoustic wave passes through the external auditory canal and reaches the tympanic membrane; the tympanic membrane transmitted these vibrations to the oval window by auditory ossicles, causing the perilymph in scala vestibuli to further pass these vibrations to the vestibular membrane and endolymph in cochlear duct. At the same time, the vibration of perilymph in scala vestibuli can be transmitted to the scala tympani through helicotrema, causing the basement membrane to resonance. Due to the different length and diameter of hearing fiber in different parts of the basement membrane results in the different frequency of acoustic wave resonance in the different parts of the basement membrane. The vibration of corresponding parts causes the HCs to contact with the tectorial membrane, the stereocilia bends, and HCs become excited to translocate the mechanical vibration into electrical excitation, which further transmit to the central auditory nerve to eventually producing the sense of hearing.

\section{Hair Cell Regeneration}

The organ of Corti harbors HCs, which are vulnerable to infections and many pharmaceutical drugs such as aminoglycoside antibodies, for example, streptomycin and neomycin, and the chemotherapeutic agent cisplatin. Most importantly, HCs can be damaged by acoustic trauma. In nonmammalian vertebrates such as birds, after ototoxic drugs or damaged by noise, the inner ear sensory HCs can regenerate spontaneously and eventually replace the damaged HCs, thus maintaining and restoring the function of sensory epithelium $[5,9]$. However, in mammals, spontaneous HC regeneration in vivo has only been identified in neonatal cochleae and also the number of regenerated HCs is quite low; as a result the hearing loss is permanent in mammals [10, 11]. It is thought that the mammalian inner ear HCs and SCs originate from the common precursor cells and some of the reported studies suggested that some SCs become HCs when the microenvironment changes, such as damage to HCs and activation of particular genes; SCs can continue to differentiate to form HCs $[12,13]$. Thus, currently some of the SCs are more commonly recognized as progenitor cells in regenerating HCs. At present, in view of the origin and regeneration of mammalian HCs, there are mainly two mechanisms of HCs regeneration from SCs, one is mitotic division of SC and the other is transdifferentiation [4-6]. In mitotic division, the SCs can divide and then their daughter cells undergo differentiating into HCs in some portions. In transdifferentiation, the SCs directly undergo phenotypic conversion and thus transdifferentiate into a $\mathrm{HC}$ without mitosis. Many studies have been done that illustrated the important factors, which are involved in the process of HC differentiation, such as Atoh1, p27Kipl, and Rb. Also, the cell signaling pathways, such as Notch, Wnt, and FGF signaling pathway, play important roles in $\mathrm{HC}$ regeneration (Figure 2).

Atohl, the bHLH differentiation factor, was relating to the formation of mechanoreceptor and photoreceptor in Drosophila $[14,15]$. During the embryonic development of mice cochlea, the upregulation of Atohl causes an increase number of HCs $[7,16]$. In the neonatal cochlea of mice, the upregulation of Atohl can activate the SCs to differentiate to form more HCs [17-19]. However, in the undamaged and mature cochleae, the differentiation capacity of SCs is significantly decreased when assessed in transgenic mice or via direct viral inoculation [20]. In consideration of the crucial role of Atohl gene during the development of HCs, various studies focused on the regulation of Atoh1 to produce HCs in the damaged and mature cochlear. It is reported that, after ototoxic injury in guinea pigs, immature HCs were regenerated through regulating the ectopic expression of Atohl, and the hearing function was rescued to a certain extent [21]. However, other studies also found that the efficacy of this approach to regenerate HCs might be limited, and the regulation time of Atohl expression after damage is dependent $[22,23]$. Moreover, it has been revealed that the $\mathrm{H} 3 \mathrm{~K} 4 \mathrm{me} 3 / \mathrm{H} 3 \mathrm{~K} 27 \mathrm{me} 3$ bivalent chromatin structure is crucial for the function of Atoh1, which is observed at the Atoh1 locus of SCs, and might give an explanation for why these cells can keep the capacity to transdifferentiate into HCs [24]. 


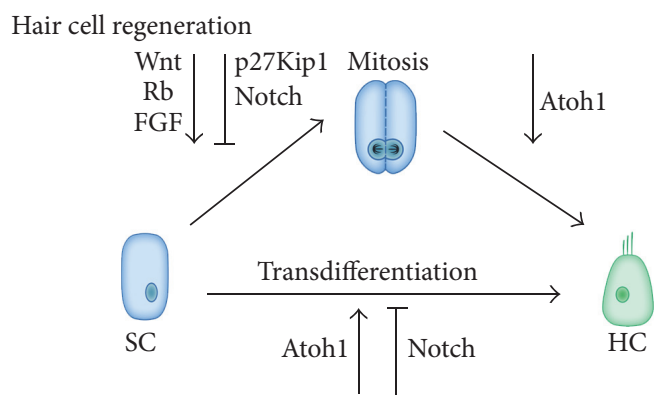

FIGURE 2

Several cyclin/cyclin-dependent-kinases (CDKs), including p27Kip1, are dynamically expressed in the sensory epithelial $[25,26]$. During the embryonic development of mammalian cochlea, the prosensory cells begin to express p27Kipl from the apex to the base [25, 26]. Disruption of p27Kipl gene in the mouse cochlea results in ongoing cell proliferation in the postnatal and adult mouse organ of Corti $[25,27]$. Although this approach partially keep the capacity of prosensory cells to proliferate, the cell overproduction will cause dysfunction in the organ of Corti, which results in hearing loss [25]. These studies indicated that the proper expression level of p27Kip1 is necessary for maintaining the normal quantity of HCs and SCs. In contrast to the nonmammals, the mammalian organ of Corti completely lacks the phenomenon through which SCs reenter cell cycle [2830]. One reason why the mature mammalian organ of Corti cannot reproduce $\mathrm{HCs}$ is because the SCs are mitotically quiescent after birth. When p27Kipl is genetically deleted in the SCs in the neonatal cochlear, these SCs proliferate but do not differentiate into HCs [31-33]. The number of mitotic cells significantly decreased in the mature cochlea when compared to that in the neonatal cochlea [31-33]. When p27Kip is deleted in the HCs of neonatal cochlea, these HCs autonomously reenter into the cell cycle and regenerate new HCs; also these newly generated HCs survived till adult age without compromising hearing function [34]. These findings revealed a new route to directly induce regeneration by renewing the proliferation capacity of surviving HCs in mammalian organ of Corti.

$\mathrm{pRb}$ is a retinoblastoma protein, which is encoded by the retinoblastoma gene Rbl. It plays a role in cell cycle exit, differentiation, and survival $[35,36]$. It has been shown that the targeted deletion of Rb1 allowed them to undergo cell cycle and become highly differentiated and functional indicating that the differentiation of the sensory epithelia and cell division are not mutually exclusive $[6,37]$. However, the proliferation due to Rb1 deletion is age dependent and eventually the cochlear HCs undergo apoptosis $[38,39]$. Moreover, the transient downregulation of Rb1 is necessary to induce proliferation in adult cochlea, also together with Rb1 deletion some other strategies such as epigenetic modifications and reprogramming need to be further studied in order to regenerate $\mathrm{HCs}$ in mature cochlea.

The Notch signaling pathway plays multiple roles during the development of mammalian cochlea. The precise formation of mosaic structure of the HC and SC is medi ated by lateral inhibition through dynamic expression of the Notch signaling pathway [40-42]. As the process of HCs differentiation begins, a prosensory cell chooses to become a $\mathrm{HC}$ or a $\mathrm{SC}$ under the precise regulation of lateral inhibition through Notch pathway. The HCs undergoing the differentiation express the Notch ligands and activate Notch signaling pathway in the neighboring SCs, thus preventing them from obtaining a $\mathrm{HC}$ fate. Eventually, the mosaic structure of $\mathrm{HC}$ and SC is formed. Moreover, in the germline deletion of the Notch ligand Jag2 or Delta-like 1 (Dll1), the $\mathrm{HC}$ number is increased at the cost of SCs [43, 44]. In a similar manner, when Notch/Jag2 and Dll1 are suppressed during early embryonic development, the prosensory cells proliferation becomes prolonged comparing with the normal control in the inner ear $[43,44]$. On the contrary, the formation of prosensory domain is prevented when the Notch receptor Notch1 is conditionally knocked out; meanwhile, there is increased number of HCs and a concomitant deceased number of SCs [43]. These findings demonstrated that the Notch pathway plays important roles in the specification of normal prosensory domain and regulates the differentiation of $\mathrm{HCs}$ in different levels through different combination of Notch ligands and receptors. Furthermore, the effects of Notch inhibition have also been explored on the regeneration process of $\mathrm{HC}$. It is reported that the SCs can transdifferentiate into $\mathrm{HCs}$ when treated with Notch inhibitor in the undamaged neonatal mammalian cochlea [45-47]. Coincidently, this pharmacological approach produces significantly less number of HCs in the damaged and mature cochlea of mammals $[48,49]$ and these newly regenerated HCs are acquired through direct transdifferentiation of SCs [45-49]. Taken together, these findings suggests that both the proliferation of SCs and HC differentiation including their coordination might require the regeneration and function recovery of the organ of Corti.

Wnt are widely expressed and evolutionary conserved in the vertebrates and invertebrates animal tissues. Wnt play important roles in several biological processes, such as development, proliferation, metabolism, and regulation of stem cells. The activation of Wnt signaling pathway through betacatenin overexpression protects $\mathrm{HCs}$ against neomycin insult [50]. When cochleae are cultured in vitro, the addition of Wnt inhibitors prevents the proliferation of prosensory cells and also the differentiation into HCs [51]. On the contrary, when supplied with Wnt signaling activators result in increased proliferation of prosensory cells and HCs [51]. These studies revealed that the canonical Wnt signaling pathway plays important roles in regulating the proliferation of prosensory cells and differentiation of HCs during cochlea development. Furthermore, when beta-catenin is ablated during cochlear development, which is a key gene of canonical Wnt signaling pathway, the proliferation of prosensory cells is significantly decreased and the large number of HCs was diminished [52]. Recent studies have found that Lgr5 positive SCs are the precursor cells with the capacity to regenerate HCs under certain conditions $[13,53]$. In the neonatal cochleae of mammals, the Wnt target gene Lgr5 is expressed in a subset of SCs (the pillar cells, inner phalangeal cells, and Deiters' cells) [54], and 
these endogenous Lgr5+ cells maintain mitotic quiescence. The expression level of Wnt signaling pathway including Lgr5 regulated via the expression of Bmil [55]. When isolated as single cells using flow cytometry and cultured in vitro, they become proliferative and converted into HC-like cells [13]. In addition, the isolated Lgr5+ SCs significantly increase the Atoh1 expression and the number of HC-like cells after the addition of Wnt signaling pathway agonist [53]. Moreover, it is reported that the proliferation capacity of the Lgr5 positive cells in the apical turn is higher than the basal turn [56]. The conditional overexpression of beta-catenin in the neonatal transgenic mouse cochlea significantly increased the percentage of proliferative supporting cells $[13,53]$. Prior study reveals that the inner pillar cells are more sensitive to the beta-catenin overexpression and can also upregulate the expression level of Atoh1 [57]. These studies suggested that the Wnt/beta-catenin signaling pathway participated in the proliferative response in the SCs of neonatal mammals and the interaction between Wnt and Notch signaling pathway is important in the inner ear $[46,58]$. More excitingly, extensive SCs proliferation followed by mitotic HCs generation can be achieved through a genetic reprogramming process involving beta-catenin activation, Notchl deletion, and Atohl overexpression. [59].

The FGF signaling pathway is important during inner ear development and morphogenesis. It is related to the induction of otic placode and the development of otic vesile [6062]. When the FGF receptor 1 (Fgfrl) is genetically deleted in the inner ear, the number of proliferative prosensory cells decreases resulting in decreased number of HCs and SCs $[63,64]$. It is reported that Fgf20, which is the candidate ligand for Fgfrl, might be the downstream target of Notch signaling pathway [42]. The addition of Ffg20 rescues the abnormal prosensory specification caused by Notch inhibition [42]. Moreover, downregulation of Fgf20 expression does not cause vestibular dysfunction, which indicates that the Fgf20 might be related to HCs specification in the cochlea. Moreover, it is identified that Fgf8 and Fgf3 are necessary for the development of pillar cells $[65,66]$. So far, the function of FGF signaling pathway on HC regeneration is explored in the utricle of chicken and lateral line of zebrafish. When SCs robustly proliferate, the expression level of Fgf20 and Fgfr3 decreases [67]. It is found that the expression level of Fgfr3 is decreased in the cochlea of chicken and the lateral line of zebrafish $[68,69]$. However, in the damaged and undamaged mammalian cochlea increased Fgfr3 expression was observed [70]. Taken together, these studies indicated that FGF signaling pathway plays an important role in the specification of prosensory cells and differentiation of HCs and SCs during development, but the function of FGF signaling on $\mathrm{HC}$ regeneration is still remain unknown.

\section{Ribbon Synapse Reforming and Reinnervation in Regenerated Hair Cells}

It is true that the regeneration of HCs is predominantly important and the pivotal issue for restoring hearing and balance function. The regeneration of synaptic connection between newly generated HCs and spiral ganglion neurons is also required. It is reported that when exposed to excessive noise, both HCs and spiral ganglion neuron are sensitive. In mammals, spiral ganglion neurons are hardly recovered from injury $[71,72]$ and the auditory nerve fibers often degenerate after ototoxic insult, including noise damage and ototoxic drugs. The process of degeneration has been revealed. At first, the unmyelinated terminal dendrites within the organ of Corti disappear (within hours to days), followed by the slow degeneration of peripheral axons in the osseous spiral lamina (within days to weeks). Then, the cell bodies in the spiral ganglion and their central axons that compose the cochlear nerve (over weeks to months and longer) degenerate in the last. Thus the regeneration of ribbon synapses and spiral ganglion neurons in combination with HCs are important for treating hearing loss.

The innervation of $\mathrm{HCs}$ is complex process. In the mammalian cochlea, the inner HCs are key component in the sound perception. The inner $\mathrm{HC}$ transmits signal to the nerve fibers of spiral ganglion neuron through transforming the mechanical signals induced by sound into electrochemical signal. On the other hand, the outer $\mathrm{HC}$ is related to the amplification of audible signals. In the auditory nervous system, there are two kinds of functional neuron population that works differently to convey sound information. In the adult mouse cochlea, there are approximately 800 inner HCs, which are exclusively innervated by 5-30 type I spiral ganglion neuron fibers. These type I spiral ganglion neurons are the main encoders of the auditory signal, which constitute almost 95 percent of the total neuron population [73-75]. In contrast, the type II spiral ganglion neurons constituting approximately 5 percent of the total neuron population innervate the approximately 2,600 outer HCs (almost 1-2 outer HCs per fiber) [76] (Figure 3). The innervation of type II spiral ganglion neuron to the outer HCs is likely to give sensory feedback as a component of the neural control loop, which includes the inhibitory olivocochlear efferent innervation of both outer $\mathrm{HC}$ and the postsynaptic region of the type I spiral ganglion neuron at the inner $\mathrm{HC}$ region. The mature organ of Corti receives extensive efferent innervation via the lateral olivocochlear (LOC) input to the boutons and dendrites of type I spiral ganglion neurons in the inner spiral plexus region and via the medial olivocochlear (MOC) bundle projection to the outer HCs [76] (Figure 3). This reorganization occurs just before the onset of hearing during the first postnatal week. There are three distinct stages in the formation and development of the afferent nerve fiber innervation to the inner and outer HCs [77]. From embryonic day 18 to postnatal day 0 , two kinds of afferent nerve fibers begin to extend and the neurite grows towards HCs. From postnatal day 0 to day 3, the neurite of these afferent fibers begins to refine to form outer spiral bundles, which innervate outer HCs. From postnatal day 3 to day 6, the neurite and synapse structure of type I spiral ganglion neuron retract towards outer $\mathrm{HCs}$ and prune to eliminate the innervation between outer HCs and type I spiral ganglion neuron, while the innervation of inner $\mathrm{HC}$ is retained by type I spiral ganglion neuron. Also, multiple factors and 


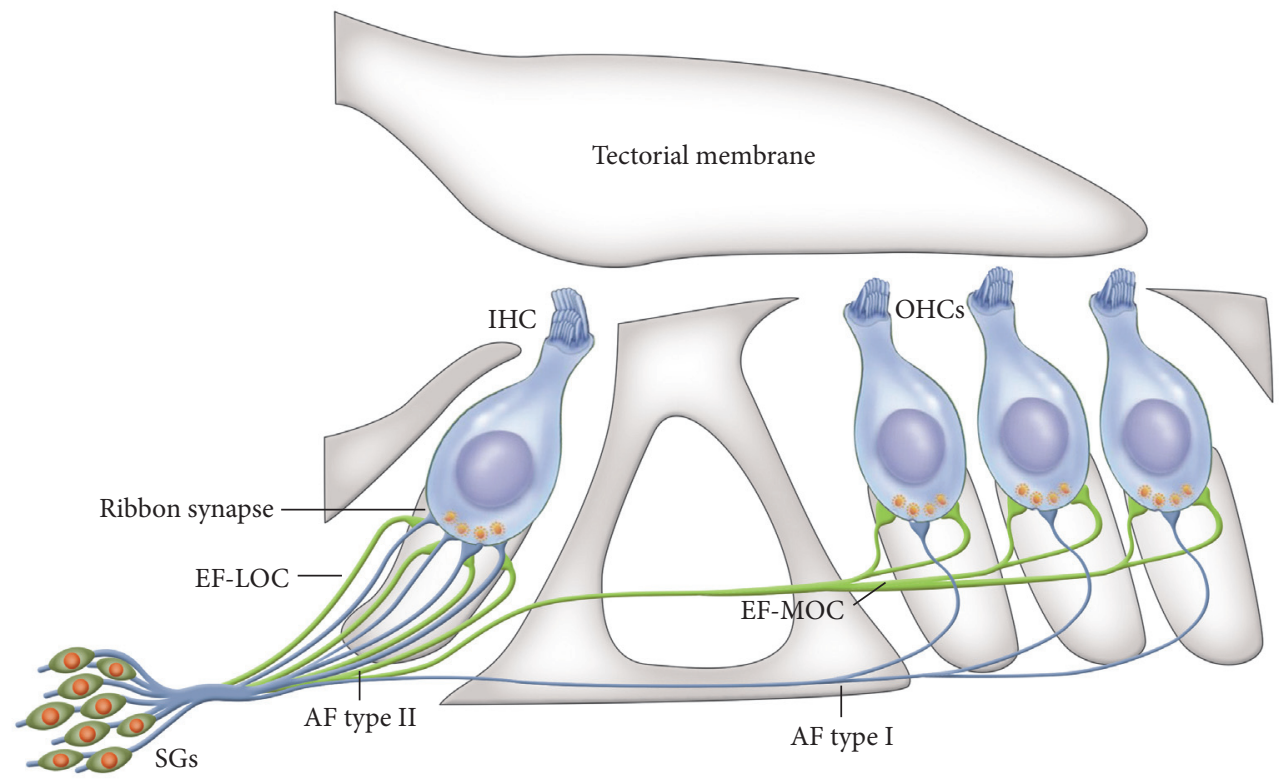

FIGURE 3: Schematic drawing of the innervation of hair cells. IHC: inner hair cell; OHCs: outer hair cells; AF: afferent fiber; EF: efferent fiber; LOC: lateral olivary complex; MOC: medial olivary complex.

signaling pathways have been studied in the development and regeneration processes in inner HC ribbon synapses, such as neurotrophins, hormonal signaling, thrombospondins, Gata3-mafb, and Foxo3 networks [78, 79].

The neurotransmission between inner HCs and type I spiral ganglion neurons and outer HCs and type II spiral ganglion neurons are conveyed by the ribbon synapses, which are crucial for the accurate encoding of acoustic information $[76,80]$. The key component of the ribbon synapse is the glutamatergic synaptic complexes, which are composed of presynaptic ribbons and postsynaptic densities. This kind of afferent ribbon synapse is capable of releasing neurotransmitter quickly and synchronously [81]. The presynaptic ribbons in the inner ear basolateral membrane was found in the opposite side of the postsynaptic glutamate receptors on the dendrite of afferent fibers. The presynaptic ribbons are settled in the active zone of HCs by electron-dense ribbon configuration. When responding to different acoustic signals, the presynaptic ribbons release multiple vesicles quickly and synchronously with high temporal resolution [82-84]. In the postsynaptic dendrite of afferent fibers, the excitatory neurotransmission is mediated by AMPA-type glutamate receptors [85].

In recent years, $\mathrm{HC}$ regeneration has made certain achievements; thus, the reinnervation of newly generated HCs and reformation of ribbon synapses are urgently needed for restoring hearing and balance function. Cochlear ribbon synapses have limited intrinsic capacity to spontaneously regenerate [86-88]. Prior study reported that when cochleae is damaged in neonatal mice, the HCs spontaneously regenerate from the SCs, but the inner cell marker vesicular glutamate transporter VGlut3 was not detected in these newly regenerated HCs [10]. When the Notchl signaling pathway overactivated to induce the ectopic HCs, the neural fiber marker Tuj1 was detected in the lateral edge of spiral ganglion neuron, while the synaptic marker synaptophysin was detected between the new HCs and neuronal cells in the spiral ganglion regions, but the synaptophysin signals adjacent to new HCs were weaker indicating that the synaptic structures among new HCs and neuronal cells were not fully mature [89]. The deletion of $\mathrm{p} 27 \mathrm{Kip} 1$ induced regeneration of new HCs and these HCs were stained with espin (stereociliary bundles), C-terminal binding protein-2 (Ctbp2; ribbon synapses), and class III beta-tubulin (Tuj1; innervating nerve fibers). However, a portion of the postnatal derived inner HCs was negative for VGlut3 (synaptic transmission) marker [34]. Deletion of p27Kip1 reforms "synaptic structure" to some extent. Although hearing function was normal in adult mice, the functional reformation of synaptic contacts still remained unclear. The ectopic expression of Atohl induced $\mathrm{HC}$ regeneration, the synaptic markers, CSP, synaptophysin, and synaptotagmin 1 detected at the basal of the newly generated HCs. Although some synaptic markers were found at the site of newly regenerated HCs and neuron contacts, the normal synaptic ribbons were still absent [18].

To achieve a better innervation of the newly generated HCs, the regeneration of ribbon synapses is predominantly important. Recently, many factors and signaling pathways have been found to play a role in promoting axonal regeneration and synapse reformation $[90,91]$. The synaptotrophic factors are the most well-known factors. The members of the neutrophin family, such as the nerve growth factor (NGF), brain-derived neurotrophic factor (BDNF), neurotrophin-3 (NT3), and neurotrophin-4/5 (NT-4/5), are participated in 
the formation of ribbon synapse and promote the synaptic regeneration process [92-95]. BDNF and its congenital receptor TrkB and the NT-3 and its congenital receptor were detected in the cochlea [96]. It has been reported that BDNF and NT-3 are critical factors for the survival of sensory neurons and the initiation of nerve fibers extending towards the sensory epithelial in the cochlea and vestibule [97]. In the neonatal inner ear of mammals, the deletion of BDNF or NT-3 causes specific loss of ribbon synapses in the cochlea and vestibule, respectively, causing hearing loss and vestibular dysfunction $[98,99]$. After ototoxic drug damage, the addition of BDNF and NT-3 promotes the reinnervation of spiral ganglion neurons in cultured cochleae and expresses the postsynaptic markers [100]. Moreover, it is likely that the NT-3 is more significant for ribbon synapses after noise exposure than BDNF [99]. Supporting cell-derived NT-3 promotes the regeneration of ribbon synapses and is helpful in the recovery of cochlear function $[78,100]$ indicating that the neurotrophins are important for the formation of postsynaptic densities and ribbon synapse regeneration after injury. Glutamate is another important synaptotrophic factor [100]. In the deafferented organ of Corti, the number of newly generated synaptic contacts at the dendrite of spiral ganglion neurons was significantly decreased in the Vglut3 deletion mice when compared to normal controls, indicating that the proper releasing of glutamate transmitter is important for the regeneration of synaptic contacts in vitro [100]. However, the in vivo role of glutamate in synaptic regeneration still remains unclear. Furthermore, the contacts generated between cultured spiral ganglion neurons and denervated HCs were evaluated and found that the postsynaptic density protein PSD-95 was immunopositive and directly facing the HC ribbons [100]. The neurotrophins, BDNF and NT-3, significantly increase the number of new synapses. In consideration of the synapse formation activities, these neurotrophins reveal a potential to promote synapse regeneration in the newly regenerated HCs.

\section{Conclusions}

In the recent years, there is growing concern about the HC regeneration and synaptic plasticity around the globe and the great achievements have been made in revealing the mechanism and strategies to recover hearing function in mammals $[10,13,59,101]$. Different levels of HC regeneration could be achieved through the regulation of factors and signaling pathways, which play important roles during the development of mammalian inner ear $[23,34,48,59]$. Synapse and nerve fiber related markers are detected around the newly regenerated HCs $[10,34,89]$. However, we are still quite far from restoring the hearing function in the damaged inner ear. The maturation and survival of newly generated HCs are still challenging. Furthermore, the maturation of reinnervation of the regenerated HCs and the function of the reformed ribbon synapse remain open to question, such as the contact between stereocilium and tectorial membrane, reorganization of the innervation of afferent Type I and Type II spiral ganglion neuron, and the integral interplay of outer hair cell based cochlear amplification. To obtain a viable treatment option for future hair cell regeneration of patients suffering from hearing loss, the understanding of reinnervation of the regenerated hair cells and the function of the reformed ribbon synapse is essential and it remains to be explored and open to question.

\section{Competing Interests}

The authors have declared that no competing interests exist.

\section{Authors' Contributions}

Xiaoling $\mathrm{Lu}$ and Yilai Shu contributed equally to this work.

\section{References}

[1] J. V. Brigande and S. Heller, "Quo vadis, hair cell regeneration?" Nature Neuroscience, vol. 12, no. 6, pp. 679-685, 2009.

[2] A. Forge, L. Li, J. T. Corwin, and G. Nevill, "Ultrastructural evidence for hair cell regeneration in the mammalian inner ear," Science, vol. 259, no. 5101, pp. 1616-1619, 1993.

[3] M. E. Warchol, P. R. Lambert, B. J. Goldstein, A. Forge, and J. T. Corwin, "Regenerative proliferation in inner ear sensory epithelia from adult guinea pigs and humans," Science, vol. 259, no. 5101, pp. 1619-1622, 1993.

[4] Y. Raphael, "Evidence for supporting cell mitosis in response to acoustic trauma in the avian inner ear," Journal of Neurocytology, vol. 21, no. 9, pp. 663-671, 1992.

[5] J. S. Stone and D. A. Cotanche, "Hair cell regeneration in the avian auditory epithelium," International Journal of Developmental Biology, vol. 51, no. 6-7, pp. 633-647, 2007.

[6] C. Sage, M. Huang, K. Karimi et al., "Proliferation of functional hair cells in vivo in the absence of the retinoblastoma protein," Science, vol. 307, no. 5712, pp. 1114-1118, 2005.

[7] S. P. Gubbels, D. W. Woessner, J. C. Mitchell, A. J. Ricci, and J. V. Brigande, "Functional auditory hair cells produced in the mammalian cochlea by in utero gene transfer," Nature, vol. 455, no. 7212, pp. 537-541, 2008.

[8] M. W. Kelley, "Regulation of cell fate in the sensory epithelia of the inner ear," Nature Reviews Neuroscience, vol. 7, no. 11, pp. 837-849, 2006.

[9] B. M. Ryals and E. W. Rubel, "Hair cell regeneration after acoustic trauma in adult coturnix quail," Science, vol. 240, no. 4860, pp. 1774-1776, 1988.

[10] B. C. Cox, R. Chai, A. Lenoir et al., "Spontaneous hair cell regeneration in the neonatal mouse cochlea in vivo," Development, vol. 141, no. 7, pp. 1599-1599, 2014.

[11] P. M. White, A. Doetzlhofer, Y. S. Lee, A. K. Groves, and N. Segil, "Mammalian cochlear supporting cells can divide and transdifferentiate into hair cells," Nature, vol. 441, no. 7096, pp. 984987, 2006.

[12] T. A. Jan, R. Chai, Z. N. Sayyid et al., "Tympanic border cells are Wnt-responsive and can act as progenitors for postnatal mouse cochlear cells," Development, vol. 140, no. 6, pp. 1196-1206, 2013.

[13] R. Chai, B. Kuo, T. Wang et al., "Wnt signaling induces proliferation of sensory precursors in the postnatal mouse cochlea," Proceedings of the National Academy of Sciences of the United States of America, vol. 109, no. 21, pp. 8167-8172, 2012.

[14] A. P. Jarman, Y. Grau, L. Y. Jan, and Y. N. Jan, "Atonal is a proneural gene that directs chordotonal organ formation in the 
Drosophila peripheral nervous system," Cell, vol. 73, no. 7, pp. 1307-1321, 1993.

[15] A. P. Jarman, E. H. Grell, L. Ackerman, L. Y. Jan, and Y. N. Jan, "Atonal is the proneural gene for Drosophila photoreceptors," Nature, vol. 369, no. 6479, pp. 398-400, 1994.

[16] C. Woods, M. Montcouquiol, and M. W. Kelley, "Math1 regulates development of the sensory epithelium in the mammalian cochlea," Nature Neuroscience, vol. 7, no. 12, pp. 1310-1318, 2004.

[17] M. C. Kelly, Q. Chang, A. Pan, X. Lin, and P. Chen, "Atoh1 directs the formation of sensory mosaics and induces cell proliferation in the postnatal mammalian cochlea in vivo," Journal of Neuroscience, vol. 32, no. 19, pp. 6699-6710, 2012.

[18] Z. Liu, J. A. Dearman, B. C. Cox et al., "Age-dependent in vivo conversion of mouse cochlear pillar and Deiters' cells to immature hair cells by Atohl ectopic expression," Journal of Neuroscience, vol. 32, no. 19, pp. 6600-6610, 2012.

[19] Z. Liu, J. Fang, J. Dearman, L. Zhang, and J. Zuo, "In vivo generation of immature inner hair cells in neonatal mouse cochleae by ectopic Atohl expression," PLoS ONE, vol. 9, no. 2, Article ID e89377, 2014.

[20] K. Kawamoto, S.-I. Ishimoto, R. Minoda, D. E. Brough, and Y. Raphael, "Mathl gene transfer generates new cochlear hair cells in mature guinea pigs in vivo," The Journal of Neuroscience, vol. 23, no. 11, pp. 4395-4400, 2003.

[21] M. Izumikawa, R. Minoda, K. Kawamoto et al., "Auditory hair cell replacement and hearing improvement by Atohl gene therapy in deaf mammals," Nature Medicine, vol. 11, no. 3, pp. 271-276, 2005.

[22] M. Izumikawa, S. A. Batts, T. Miyazawa, D. L. Swiderski, and Y. Raphael, "Response of the flat cochlear epithelium to forced expression of Atohl," Hearing Research, vol. 240, no. 1-2, pp. 5256, 2008.

[23] P. J. Atkinson, A. K. Wise, B. O. Flynn, B. A. Nayagam, and R. T. Richardson, "Hair cell regeneration after ATOH1 gene therapy in the cochlea of profoundly deaf adult guinea pigs," PLOS ONE, vol. 9, no. 7, Article ID e102077, 2014.

[24] Z. P. Stojanova, T. Kwan, and N. Segil, "Epigenetic regulation of Atohl guides hair cell development in the mammalian cochlea," Development, vol. 143, article 1632, 2016.

[25] P. Chen and N. Segil, "p27(Kip1) links cell proliferation to morphogenesis in the developing organ of Corti," Development, vol. 126, no. 8, pp. 1581-1590, 1999.

[26] R. J. Ruben, "Development of the inner ear of the mouse: a radioautographic study of terminal mitoses," Acta OtoLaryngologica, supplement 220, pp. 221-244, 1967.

[27] H. Lowenheim, D. N. Furness, J. Kil et al., "Gene disruption of p27(Kip1) allows cell proliferation in the postnatal and adult organ of corti," Proceedings of the National Academy of Sciences of the United States of America, vol. 96, no. 7, pp. 4084-4088, 1999.

[28] J. A. Harris, A. G. Cheng, L. L. Cunningham, G. MacDonald, D. W. Raible, and E. W. Rubel, "Neomycin-induced hair cell death and rapid regeneration in the lateral line of zebrafish (Danio rerio)," Journal of the Association for Research in Otolaryngology, vol. 4, no. 2, pp. 219-234, 2003.

[29] P. P. Hernández, F. A. Olivari, A. F. Sarrazin, P. C. Sandoval, and M. L. Allende, "Regeneration in zebrafish lateral line neuromasts: expression of the neural progenitor cell marker sox2 and proliferation-dependent and-independent mechanisms of hair cell renewal," Developmental Neurobiology, vol. 67, no. 5, pp. 637-654, 2007.
[30] J. I. Matsui, E. C. Oesterle, J. S. Stone, and E. W. Rubel, "Characterization of damage and regeneration in cultured avian utricles," Journal of the Association for Research in Otolaryngology, vol. 1, no. 1, pp. 46-63, 2000.

[31] Z. Liu, B. J. Walters, T. Owen et al., "Regulation of $\mathrm{p} 27^{\text {Kip } 1}$ by Sox 2 maintains quiescence of inner pillar cells in the murine auditory sensory epithelium," Journal of Neuroscience, vol. 32, no. 31, pp. 10530-10540, 2012.

[32] J. C. Maass, F. A. Berndt, J. Cánovas, and M. Kukuljan, “P27Kip1 knockdown induces proliferation in the organ of Corti in culture after efficient shRNA lentiviral transduction," Journal of the Association for Research in Otolaryngology, vol. 14, no. 4, pp. 495-508, 2013.

[33] E. C. Oesterle, W. M. Chien, S. Campbell et al., "p27(Kip1) is required to maintain proliferative quiescence in the adult cochlea and pituitary," Cell Cycle, vol. 10, no. 8, pp. 1237-1248, 2011.

[34] B. J. Walters, Z. Liu, M. Crabtree, E. Coak, B. C. Cox, and J. Zuo, "Auditory hair cell-specific deletion of p27Kipl in postnatal mice promotes cell-autonomous generation of new hair cells and normal hearing," The Journal of Neuroscience, vol. 34, no. 47, pp. 15751-15763, 2014.

[35] M. Classon and E. Harlow, "The retinoblastoma tumour suppressor in development and cancer," Nature Reviews Cancer, vol. 2, no. 12, pp. 910-917, 2002.

[36] M. M. Lipinski and T. Jacks, "The retinoblastoma gene family in differentiation and development," Oncogene, vol. 18, no. 55, pp. 7873-7882, 1999.

[37] C. Sage, M. Huang, M. A. Vollrath et al., "Essential role of retinoblastoma protein in mammalian hair cell development and hearing," Proceedings of the National Academy of Sciences of the United States of America, vol. 103, no. 19, pp. 7345-7350, 2006.

[38] J. Mantela, Z. Jiang, J. Ylikoski, B. Fritzsch, E. Zacksenhaus, and U. Pirvola, "The retinoblastoma gene pathway regulates the postmitotic state of hair cells of the mouse inner ear," Development, vol. 132, no. 10, pp. 2377-2388, 2005.

[39] Y. Yu, T. Weber, T. Yamashita et al., "In vivo proliferation of postmitotic cochlear supporting cells by acute ablation of the retinoblastoma protein in neonatal mice," Journal of Neuroscience, vol. 30, no. 17, pp. 5927-5936, 2010.

[40] R. Brooker, K. Hozumi, and J. Lewis, "Notch ligands with contrasting functions: Jagged1 and Deltal in the mouse inner ear," Development, vol. 133, no. 7, pp. 1277-1286, 2006.

[41] A. E. Kiernan, J. Xu, and T. Gridley, "The Notch ligand JAG1 is required for sensory progenitor development in the mammalian inner ear," PLoS Genetics, vol. 2, no. 1, article no. e4, 2006.

[42] V. Munnamalai, T. Hayashi, and O. Bermingham-McDonogh, "Notch prosensory effects in the mammalian cochlea are partially mediated by Fgf20," Journal of Neuroscience, vol. 32, no. 37, pp. 12876-12884, 2012.

[43] A. E. Kiernan, R. Cordes, R. Kopan, A. Gossler, and T. Gridley, "The Notch ligands DLL1 and JAG2 act synergistically to regulate hair cell development in the mammalian inner ear," Development, vol. 132, no. 19, pp. 4353-4362, 2005.

[44] P. J. Lanford, Y. Lan, R. Jiang et al., "Notch signalling pathway mediates hair cell development in mammalian cochlea," Nature Genetics, vol. 21, no. 3, pp. 289-292, 1999.

[45] A. Doetzlhofer, M. L. Basch, T. Ohyama, M. Gessler, A. K. Groves, and N. Segil, "Hey2 regulation by fgf provides a Notchindependent mechanism for maintaining pillar cell fate in the 
organ of corti," Developmental Cell, vol. 16, no. 1, pp. 58-69, 2009.

[46] W. Li, J. Wu, J. Yang et al., "Notch inhibition induces mitotically generated hair cells in mammalian cochleae via activating the Wnt pathway," Proceedings of the National Academy of Sciences of the United States of America, vol. 112, no. 1, pp. 166-171, 2015.

[47] N. Yamamoto, K. Tanigaki, M. Tsuji, D. Yabe, J. Ito, and T. Honjo, "Inhibition of Notch/RBP-J signaling induces hair cell formation in neonate mouse cochleas," Journal of Molecular Medicine, vol. 84, no. 1, pp. 37-45, 2006.

[48] K. Mizutari, M. Fujioka, M. Hosoya et al., "Notch inhibition induces cochlear hair cell regeneration and recovery of hearing after acoustic trauma," Neuron, vol. 77, no. 1, pp. 58-69, 2013.

[49] Y. Tona, K. Hamaguchi, M. Ishikawa et al., "Therapeutic potential of a gamma-secretase inhibitor for hearing restoration in a guinea pig model with noise-induced hearing loss," $B M C$ Neuroscience, vol. 15, article 66, 2014.

[50] L. Liu, Y. Chen, J. Qi et al., "Wnt activation protects against neomycin-induced hair cell damage in the mouse cochlea," Cell Death and Disease, vol. 7, no. 3, article e2136, 2016.

[51] B. E. Jacques, C. Puligilla, R. M. Weichert et al., "A dual function for canonical Wnt $/ \beta$-catenin signaling in the developing mammalian cochlea," Development, vol. 139, no. 23, pp. 4395-4404, 2012.

[52] F. Shi, L. Hu, B. E. Jacques, J. F. Mulvaney, A. Dabdoub, and A. S. B. Edge, " $\beta$-catenin is required for hair-cell differentiation in the cochlea," Journal of Neuroscience, vol. 34, no. 19, pp. 6470-6479, 2014.

[53] F. Shi, J. S. Kempfle, and A. S. B. Edge, "Wnt-responsive Lgr5expressing stem cells are hair cell progenitors in the cochlea," Journal of Neuroscience, vol. 32, no. 28, pp. 9639-9684, 2012.

[54] R. Chai, A. Xia, T. Wang et al., "Dynamic expression of Lgr5, a Wnt target gene, in the developing and mature mouse cochlea," Journal of the Association for Research in Otolaryngology: JARO, vol. 12, no. 4, pp. 455-469, 2011.

[55] X. Lu, S. Sun, J. Qi et al., "Bmil regulates the proliferation of cochlear supporting cells via the canonical Wnt signaling pathway," Molecular Neurobiology, pp. 1-14, 2016.

[56] M. Waqas, L. Guo, S. Zhang et al., "Characterization of Lgr5+ progenitor cell transcriptomes in the apical and basal turns of the mouse cochlea," Oncotarget, vol. 7, no. 27, pp. 41123-41141, 2016.

[57] F. Shi, L. Hu, and A. S. B. Edge, "Generation of hair cells in neonatal mice by $\beta$-catenin overexpression in Lgr5-positive cochlear progenitors," Proceedings of the National Academy of Sciences of the United States of America, vol. 110, no. 34, pp. 13851-13856, 2013.

[58] M. Waqas, S. Zhang, Z. He, M. Tang, and R. Chai, "Role of Wnt and Notch signaling in regulating hair cell regeneration in the cochlea," Frontiers of Medicine, vol. 10, no. 3, pp. 237-249, 2016.

[59] W. Ni, C. Lin, L. Guo et al., "Extensive supporting cell proliferation and mitotic hair cell generation by in vivo genetic reprogramming in the neonatal mouse cochlea," Journal of Neuroscience, vol. 36, no. 33, pp. 8734-8745, 2016.

[60] U. Pirvola, B. Spencer-Dene, L. Xing-Qun et al., "FGF/FGFR2(IIIb) signaling is essential for inner ear morphogenesis," The Journal of Neuroscience, vol. 20, no. 16, pp. 6125-6134, 2000.

[61] T. Schimmang, "Expression and functions of FGF ligands during early otic development," International Journal of Developmental Biology, vol. 51, no. 6-7, pp. 473-481, 2007.
[62] T. J. Wright and S. L. Mansour, "Fgf3 and Fgf10 are required for mouse otic placode induction," Development, vol. 130, no. 15, pp. 3379-3390, 2003.

[63] K. Ono, T. Kita, S. Sato et al., "FGFR1-Frs2/3 signalling maintains sensory progenitors during inner ear hair cell formation," PLOS Genetics, vol. 10, no. 1, Article ID e1004118, 2014.

[64] U. Pirvola, J. Ylikoski, R. Trokovic, J. M. Hébert, S. K. McConnell, and J. Partanen, "FGFR1 is required for the development of the auditory sensory epithelium," Neuron, vol. 35, no. 4, pp. 671-680, 2002.

[65] J. S. Colvin, B. A. Bohne, G. W. Harding, D. G. McEwen, and D. M. Ornitz, "Skeletal overgrowth and deafness in mice lacking fibroblast growth factor receptor 3," Nature Genetics, vol. 12, no. 4, pp. 390-397, 1996.

[66] B. E. Jacques, M. E. Montcouquiol, E. M. Layman, M. Lewandoski, and M. W. Kelley, "Fgf8 induces pillar cell fate and regulates cellular patterning in the mammalian cochlea," Development, vol. 134, no. 16, pp. 3021-3029, 2007.

[67] Y.-C. Ku, N. A. Renaud, R. A. Veile et al., "The transcriptome of utricle hair cell regeneration in the avian inner ear," The Journal of Neuroscience, vol. 34, no. 10, pp. 3523-3535, 2014.

[68] O. Bermingham-McDonogh, J. S. Stone, T. A. Reh, and E. W. Rubel, "FGFR3 expression during development and regeneration of the chick inner ear sensory epithelia," Developmental Biology, vol. 238, no. 2, pp. 247-259, 2001.

[69] L. Jiang, A. Romero-Carvajal, J. S. Haug, C. W. Seidel, and T. Piotrowski, "Gene-expression analysis of hair cell regeneration in the zebrafish lateral line," Proceedings of the National Academy of Sciences of the United States of America, vol. 111, no. 14, pp. E1383-E1392, 2014.

[70] U. Pirvola, Y. Cao, C. Oellig, Z. Suoqiang, R. F. Pettersson, and J. Ylikoski, "The site of action of neuronal acidic fibroblast growth factor is the organ of Corti of the rat cochlea," Proceedings of the National Academy of Sciences of the United States of America, vol. 92, no. 20, pp. 9269-9273, 1995.

[71] K. Liu, X. Jiang, C. Shi et al., "Cochlear inner hair cell ribbon synapse is the primary target of ototoxic aminoglycoside stimuli," Molecular Neurobiology, vol. 48, no. 3, pp. 647-654, 2013.

[72] L. Shi, K. Liu, H. Wang et al., "Noise induced reversible changes of cochlear ribbon synapses contribute to temporary hearing loss in mice," Acta Oto-Laryngologica, vol. 135, no. 11, pp. 10931102, 2015.

[73] E. M. Keithley and M. L. Feldman, "Hair cell counts in an agegraded series of rat cochleas," Hearing Research, vol. 8, no. 3, pp. 249-262, 1982.

[74] N. B. Slepecky and Y. Ogata, "Immunohistochemical labeling of inner ear tissues embedded in polyethylene glycol 4000comparative study with araldite and unicryl embedded sections," Nippon Jibiinkoka Gakkai kaiho, vol. 99, no. 3, pp. 361$369,1996$.

[75] H. Burda and M. Branis, "Postnatal development of the organ of Corti in the wild house mouse, laboratory mouse, and their hybrid," Hearing Research, vol. 36, no. 1, pp. 97-105, 1988.

[76] W. B. Warr and J. J. Guinan Jr., "Efferent innervation of the organ of corti: two separate systems," Brain Research, vol. 173, no. 1, pp. $152-155,1979$.

[77] L. C. Huang, P. R. Thorne, G. D. Housley, and J. M. Montgomery, "Spatiotemporal definition of neurite outgrowth, refinement and retraction in the developing mouse cochlea," Development, vol. 134, no. 16, pp. 2925-2933, 2007. 
[78] Q. Wang and S. H. Green, "Functional role of neurotrophin-3 in synapse regeneration by spiral ganglion neurons on inner hair cells after excitotoxic trauma in vitro," Journal of Neuroscience, vol. 31, no. 21, pp. 7938-7949, 2011.

[79] W. Singer, R. Panford-Walsh, and M. Knipper, "The function of BDNF in the adult auditory system," Neuropharmacology, vol. 76, pp. 719-728, 2014.

[80] C. Weisz, E. Glowatzki, and P. Fuchs, "The postsynaptic function of type II cochlear afferents," Nature, vol. 461, no. 7267, pp. 1126-1129, 2009.

[81] S. Safieddine, A. El-Amraoui, and C. Petit, "The auditory hair cell ribbon synapse: from assembly to function," Annual Review of Neuroscience, vol. 35, pp. 509-528, 2012.

[82] P. A. Fuchs, "Time and intensity coding at the hair cell's ribbon synapse," Journal of Physiology, vol. 566, no. 1, pp. 7-12, 2005.

[83] D. Khimich, R. Nouvian, R. Pujol et al., "Hair cell synaptic ribbons are essential for synchronous auditory signalling," Nature, vol. 434, no. 7035, pp. 889-894, 2005.

[84] R. Nouvian, D. Beutner, T. D. Parsons, and T. Moser, "Structure and function of the hair cell ribbon synapse," Journal of Membrane Biology, vol. 209, no. 2-3, pp. 153-165, 2006.

[85] R. T. Fremeau Jr., S. Voglmaier, R. P. Seal, and R. H. Edwards, "VGLUTs define subsets of excitatory neurons and suggest novel roles for glutamate," Trends in Neurosciences, vol. 27, no. 2, pp. 98-103, 2004.

[86] S. F. Maison, H. Usubuchi, and M. Charles Liberman, "Efferent feedback minimizes cochlear neuropathy from moderate noise exposure," Journal of Neuroscience, vol. 33, no. 13, pp. 5542-5552, 2013.

[87] R. Martinez-Monedero, C. E. Corrales, M. P. Cuajungco, S. Heller, and A. S. B. Edge, "Reinnervation of hair cells by auditory neurons after selective removal of spiral ganglion neurons," Journal of Neurobiology, vol. 66, no. 4, pp. 319-331, 2006.

[88] M. Matsumoto, T. Nakagawa, K. Kojima, T. Sakamoto, F. Fujiyama, and J. Ito, "Potential of embryonic stem cell-derived neurons for synapse formation with auditory hair cells," Journal of Neuroscience Research, vol. 86, no. 14, pp. 3075-3085, 2008.

[89] Z. Liu, T. Owen, J. Fang, and J. Zuo, "Overactivation of notch1 signaling induces ectopic hair cells in the mouse inner ear in an age-dependent manner," PLoS ONE, vol. 7, no. 3, Article ID e34123, 2012.

[90] K. A. Deyst, J. Ma, J. R. Fallon, R. G. Dacey, and C. J. Hodge Jr., "Agrin: toward a molecular understanding of synapse regeneration," Neurosurgery, vol. 37, no. 1, pp. 71-77, 1995.

[91] P. R. Gordon-Weeks and A. E. Fournier, "Neuronal cytoskeleton in synaptic plasticity and regeneration," Journal of Neurochemistry, vol. 129, no. 2, pp. 206-212, 2014.

[92] L. T. Alto, L. A. Havton, J. M. Conner, E. R. Hollis II, A. Blesch, and M. H. Tuszynski, "Chemotropic guidance facilitates axonal regeneration and synapse formation after spinal cord injury," Nature Neuroscience, vol. 12, no. 9, pp. 1106-1113, 2009.

[93] L.-X. Deng, P. Deng, Y. Ruan et al., "A novel growth-promoting pathway formed by GDNF-overexpressing Schwann cells promotes propriospinal axonal regeneration, synapse formation, and partial recovery of function after spinal cord injury," Journal of Neuroscience, vol. 33, no. 13, pp. 5655-5667, 2013.

[94] K. J. M. Marler, E. Becker-Barroso, A. Martínez et al., "A TrkB/EphrinA interaction controls retinal axon branching and synaptogenesis," The Journal of Neuroscience, vol. 28, no. 48, pp. $12700-12712,2008$.
[95] H. Park and M.-M. Poo, "Neurotrophin regulation of neural circuit development and function," Nature Reviews Neuroscience, vol. 14, no. 1, pp. 7-23, 2013.

[96] D. Ramekers, H. Versnel, W. Grolman, and S. F. L. Klis, "Neurotrophins and their role in the cochlea," Hearing Research, vol. 288, no. 1-2, pp. 19-33, 2012.

[97] B. Fritzsch, L. Tessarollo, E. Coppola, and L. F. Reichardt, "Neurotrophins in the ear: their roles in sensory neuron survival and fiber guidance," Progress in Brain Research, vol. 146, pp. 265-278, 2004.

[98] M. E. Gómez-Casati, J. C. Murtie, C. Rio, K. Stankovic, M. C. Liberman, and G. Corfas, "Nonneuronal cells regulate synapse formation in the vestibular sensory epithelium via erbB-dependent BDNF expression," Proceedings of the National Academy of Sciences of the United States of America, vol. 107, no. 39, pp. 17005-17010, 2010.

[99] G. Wan, M. E. Gómez-Casati, A. R. Gigliello, M. Charles Liberman, and G. Corfas, "Neurotrophin-3 regulates ribbon synapse density in the cochlea and induces synapse regeneration after acoustic trauma," eLife, vol. 3, Article ID e03564, 2014.

[100] M. Tong, A. Brugeaud, and A. S. B. Edge, "Regenerated synapses between postnatal hair cells and auditory neurons," Journal of the Association for Research in Otolaryngology, vol. 14, no. 3, pp. 321-329, 2013.

[101] L. L. Cunningham and D. L. Tucci, "Restoring synaptic connections in the inner ear after noise damage," The New England Journal of Medicine, vol. 372, no. 2, pp. 181-182, 2015. 

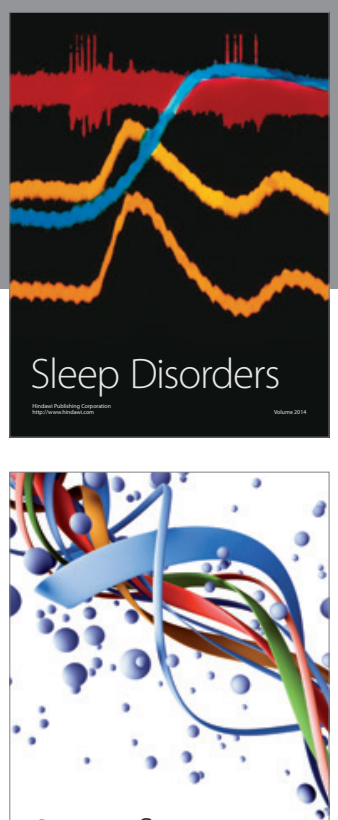

Scientifica
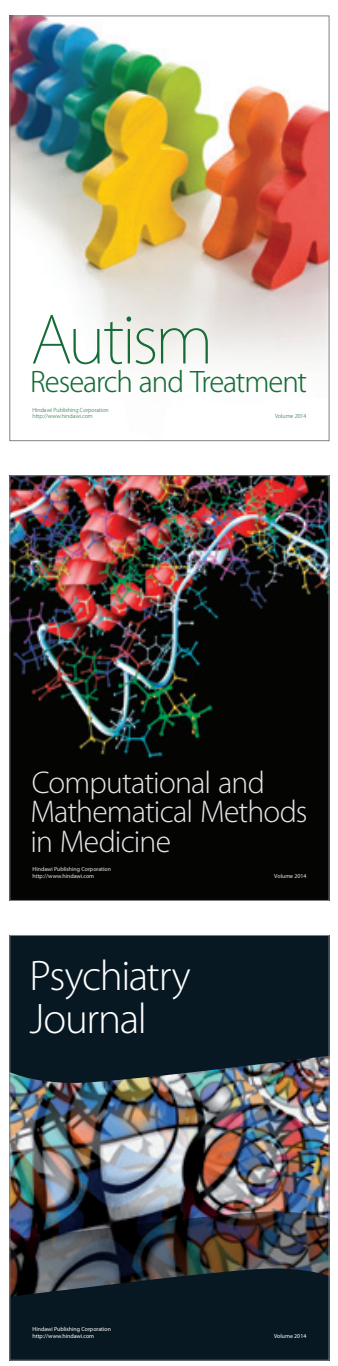
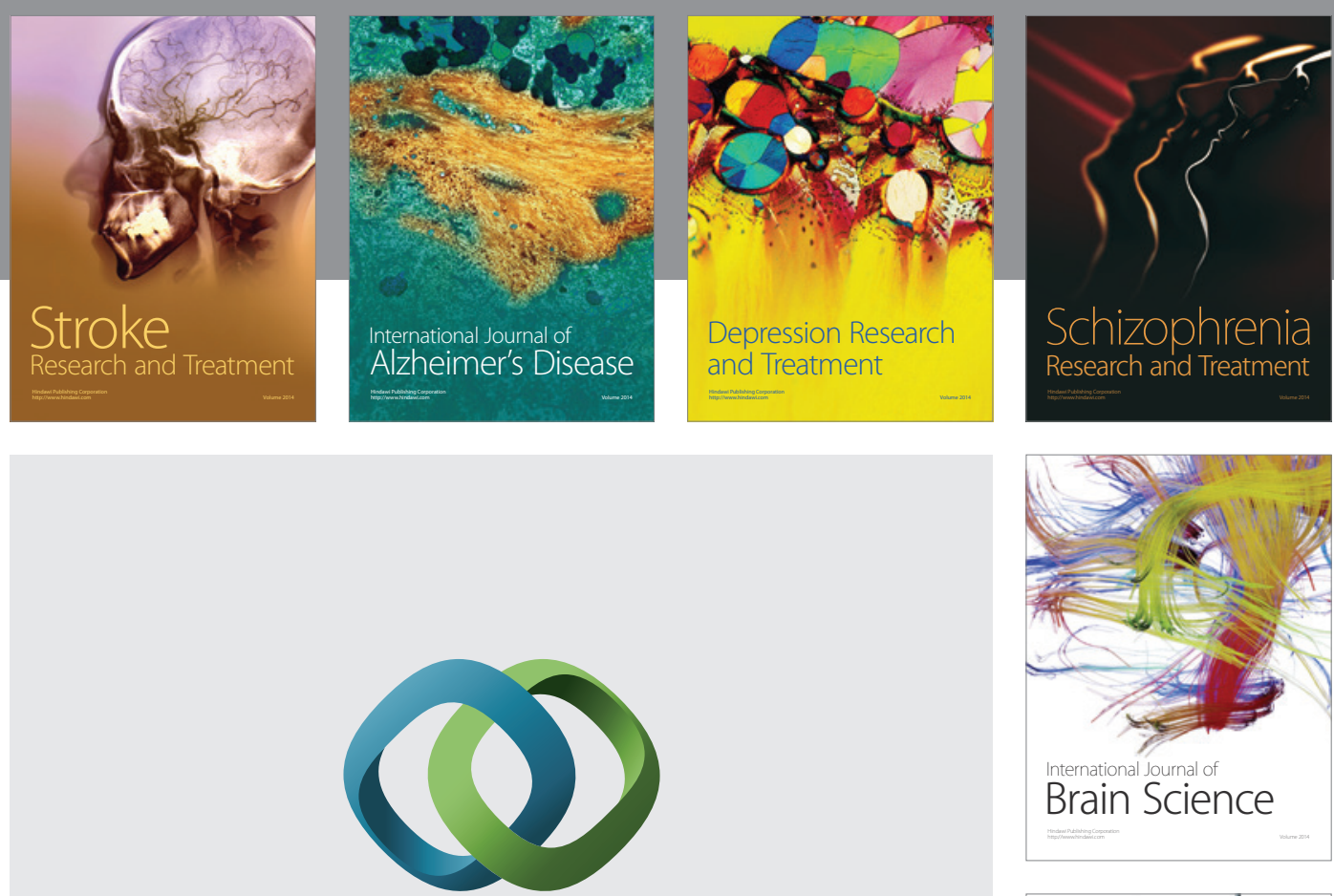

\section{Hindawi}

Submit your manuscripts at

http://www.hindawi.com
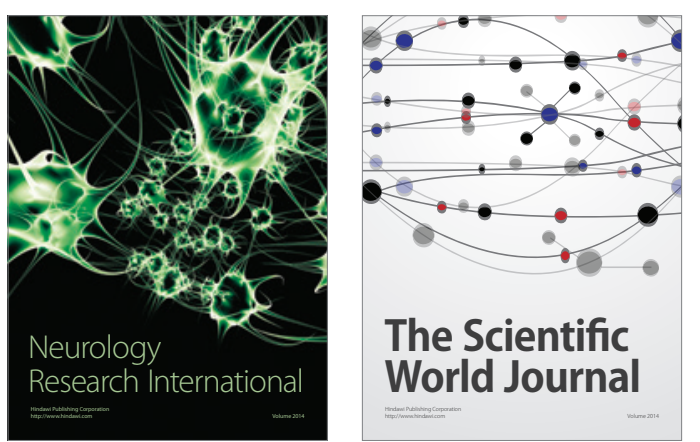

The Scientific World Journal

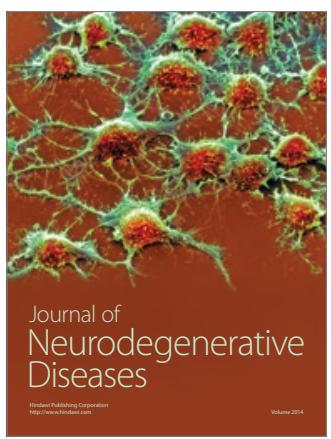

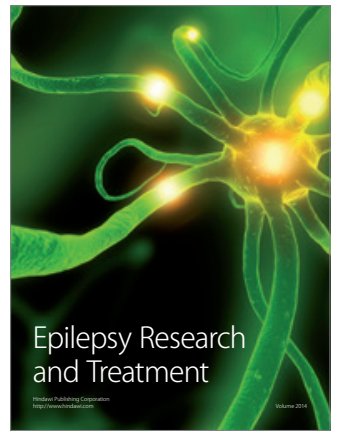

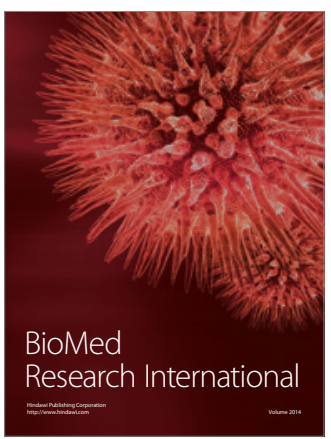

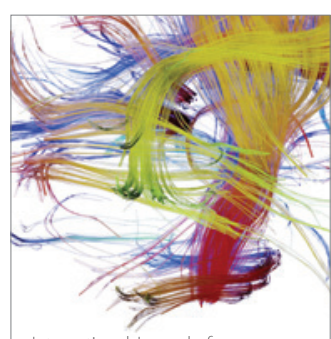

Brain Science

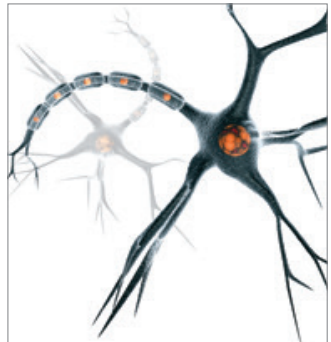

Neural Plasticity
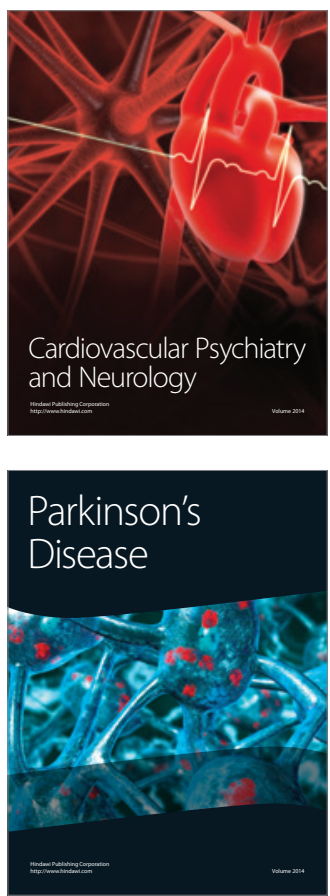